\title{
Anticancer, Anti-Inflammatory, and Analgesic Activities of Synthesized 2-(Substituted phenoxy) Acetamide Derivatives
}

\author{
Priyanka Rani, ${ }^{1}$ Dilipkumar Pal, ${ }^{2}$ Rahul Rama Hegde, ${ }^{3}$ and Syed Riaz Hashim ${ }^{4}$ \\ ${ }^{1}$ Department of Chemistry, School of Sciences, IFTM University, Moradabad, Uttar Pradesh, India \\ ${ }^{2}$ Department of Pharmaceutical Sciences, Guru Ghasidas Vishwavidyalaya (A Central University), Koni, Bilaspur, \\ Chhattisgarh 495 009, India \\ ${ }^{3}$ Department of Pharmaceutics, School of Pharmaceutical Sciences, IFTM University, Moradabad, Uttar Pradesh, India \\ ${ }^{4}$ Department of Chemistry, School of Pharmaceutical Sciences, IFTM University, Moradabad, Uttar Pradesh, India
}

Correspondence should be addressed to Priyanka Rani; skiftm@gmail.com

Received 27 February 2014; Revised 22 July 2014; Accepted 29 July 2014; Published 14 August 2014

Academic Editor: Raymond L. Konger

Copyright (C) 2014 Priyanka Rani et al. This is an open access article distributed under the Creative Commons Attribution License, which permits unrestricted use, distribution, and reproduction in any medium, provided the original work is properly cited.

The aphorism was to develop new chemical entities as potential anticancer, anti-inflammatory, and analgesic agents. The Leuckart synthetic pathway was utilized in development of novel series of 2-(substituted phenoxy)-N-(1-phenylethyl)acetamide derivatives. The compounds containing 1-phenylethylamine as basic moiety attached to substituted phenols were assessed for their anticancer activity against MCF-7 (breast cancer), SK-N-SH (neuroblastoma), anti-inflammatory activity, and analgesic activity. These investigations revealed that synthesized products $3 \mathbf{a}-\mathbf{j}$ with halogens on the aromatic ring favors as the anticancer and antiinflammatory activity. Among all, compound 3c N-(1-(4-chlorophenyl)ethyl)-2-(4-nitrophenoxy)acetamide exhibited anticancer, anti-inflammatory, and analgesic activities. In conclusion, $3 \mathrm{c}$ may have potential to be developed into a therapeutic agent.

\section{Introduction}

In the past decade, numerous advances have taken place in the understanding of pathogenesis of cancer and its relationship with inflammation. To date the research work on this ground is substantial, but it still lacks clinical accomplishment, with the existing development facade over the clinical exploitation of drugs with dual acting cyclooxygenase- 2 (COX-2) inhibition and antiproliferative potency [1]. Whenever cells in the body are allowed to divide uncontrollably and to metastasize, this results in the formation of cancer. Large scale of studies indicates that overexpression of COX2 strongly appeared in human breast carcinomas approximately $40 \%$ and in colorectal carcinomas approximately $60 \%$. In particular, COX-2 is found during overexpression of human epidermal growth factor receptor 2 (HER2/neu). The amplification of this oncogene plays an important role in the development of some aggressive types of breast cancer. COX-2 is also been reported in early tumors by stromal cells [2] and in larger tumors by dysplastic epithelium [3]. It has been proved that various inflammatory cells, cytokines, chemokines, and enzymes facilitate the development of cancers from inflammation [4]. A few COX-2/LOX2 (lipooxygenase-2) inhibitors such as sulindac, celecoxib, licofelone, and aspirin analogues have also been reported as suppressors of malignant growth of cells in vitro and in vivo [5] and Alzheimer's disease [6]. This designates that there is an unfamiliar association involving cancer and inflammation [7].

Nowadays no data are available concerning the potential use of anticancer agents as COX/LOX inhibitors. Recent molecular targets for the treatment of cancer are the relation between arachidonic acid (AA) and carcinogenesis because of the regulation of AA by two enzymes, cyclooxygenases (COXs) and lipoxygenases (LOXs). Prostaglandin $\mathrm{E}_{2}\left(\mathrm{PGE}_{2}\right)$, the main product of COX-2, is found in high concentration in tumor cells [8] and is synthesized by various human breast cancer cell lines. The goal of this research was to achieve a novel series of agents that could have potent anticancer efficacy in association with the suppression of COX/LOX pathways. 
<smiles>CC(=O)Oc1ccc(NC(=O)COc2ccc(C)cc2C)cc1</smiles>

1a<smiles>[R]c1cccc(NC(=O)COc2ccc([N+](=O)[O-])c(F)c2)c1</smiles>

$1 b$<smiles>[R]c1cccc(NC(=O)COc2ccc(Cl)cc2Cl)c1</smiles>

$1 \mathrm{c}$

Figure 1: The commonly used structures (1a, $\mathbf{l b}$ 2-Phenoxy-N-phenylacetamide core nucleus with antimycobacterial activity) (1c phenoxy$\mathrm{N}$-phenylacetamide compounds with potent $\mathrm{P}$-gp inhibitor activities).

In the present paper a novel and efficient strategy has been developed to synthesize $\mathrm{N}$-(1-(4-chlorophenyl) ethyl)-2-(substituted phenoxy)acetamide derivatives and 2(substituted phenoxy)-N-(1-(p-tolyl)ethyl)acetamide derivatives with excellent yields. This synthetic pathway was established by the Leuckart reaction. The Leuckart reaction is a process for the reductive amination of aldehydes and ketones by formamide, ammonium formate, or formic acid with formamide [9]. This reaction has been used for the aromatic compounds, but very little work has been reported for the synthesis of aliphatic compounds (synthesis of 2-heptylamine, propylamine, and isopropylamine) [10]. Apart from the aliphatic compounds, 1,4-benzodiazepines were synthesized by the Leuckart Wallach reaction containing the benzodiazepine scaffold and were originated with various biological activities (benzodiazepine family) comprised mainly of central nervous system (CNS) suppressant due to its anxiolytic, anticonvulsant, sedative, and muscle relaxant activities. It is used in various marketed drugs such as alprazolam, bromazepam, chlorazepate, and valium [11]. 1,4-Benzodiazepines also demonstrate therapeutic activities and are used as antibiotics [12], antiulcers [13], and anti-HIV agents [14]. They are also used as farnesyltransferase inhibitors [15]. Compounds with 2-phenoxy-Nphenylacetamide core nucleus as in Figure 1 (1a and $\mathbf{1 b}$ ) have shown remarkable research and demonstrated a variety of biological activities such as antimycobacterial [16], antiparasitic [17], antiviral [18], and anticancer [19] activities [20-23]. These compounds are also reported with inhibition of Pgp efflux transporters which are beneficial in the treatment of multidrug resistant strains of cancer cells. The phenoxy-Nphenylacetamide compounds are reported to be less toxic and more effective as that of potent P-gp inhibitors (coumarin analogues, 2-adamantyl analogues); see Figure 1 (1c) [24]. Now, we are reporting a new series of agents that have potent anticancer activities. These agents possibly may act upon COX/LOX pathways which may be included as a part of our future research program.

These compounds were further biologically evaluated for analgesic activity, and few of them showed activity. For the development of novel anticancer agents with lower toxic effect and higher efficiency, we carried out high throughput screening, which resulted in the discovery of titled compounds. Now, we report structure activity relationships and biological assessment of the titled compounds.

\section{Experiment}

2.1. General Considerations. All research chemicals were purchased from CDH (Central Drug House P. Ltd., New Delhi, India) and used as such for the reactions. These were used without further purification. Purification of the synthesized compounds was carried out by the recrystallization with appropriate solvent in case of solids but by distillation in case of liquids. Purity of the compounds and completion of reactions were monitored by thin layer chromatography (TLC) using silica gel G as the stationary phase and mobile phases used were $n$-hexane : ethyl acetate $(1: 1)$. Spots were visualized by exposure to iodine vapour. Melting points were determined in open capillaries on Thomas Hoover apparatus and are uncorrected. IR spectra were recorded on a Shimadzu IR-435 spectrophotometer using $\mathrm{KBr}$ pellets and $1 \mathrm{H}-\mathrm{NMR}$ spectra were recorded on a Bruker $400 \mathrm{MHz}$ spectrometer (Bruker Corporation, Massachusetts, USA) instrument using 
tetramethylsilane (TMS) as an internal standard and DMSOd6 as a solvent. Mass spectra were recorded on Micromass Q-Tof Micro (Waters Corporation Massachusetts, USA). Chemical shifts are given in parts per million (ppm). The anticancer activity was carried out at Tata Memorial Hospital, Mumbai. The anti-inflammatory and analgesic screenings are carried out at pharmacology laboratory of College of Pharmacy IFTM University, Moradabad. The anti-inflammatory activity was carried out using digital plethysmometer (Orchid Scientific, Maharashtra, India). The analgesic activity was carried out by Eddy hot plate method using analgesiometer (Sanghmeshwar International, Ambala, India). All the animal experiments were approved by Institutional Animal Ethical Committee (IAEC).

\subsection{Synthesis}

2.2.1. General Method for the Synthesis of 1-(4-Chlorophenyl)ethanamine (1a) and 1-(p-Tolyl)ethanamine (1b) from 1-(4-Chlorophenyl)ethanone and 1-(p-Tolyl)ethanone, Respectively. This procedure of the Leuckart reaction was used for the preparation of amines from ketones. Ammonium carbonate ( $215 \mathrm{gm}, 4 \mathrm{mols}$ ) was placed in a 1 litre three-necked round bottom flask, which was fitted with a thermometer, a dropping funnel, and a bent tube attached for distillation to a short condenser. Formic acid $(98 \%, 109 \mathrm{~mL})$ was taken in the dropping funnel and added dropwise. When the reaction subsided, the mixture was heated slowly until the temperature of the reaction increased to about $165^{\circ} \mathrm{C}$. The ketone $(1 \mathrm{~mol})$ was added in one lot and the temperature was slowly raised to $180-185^{\circ} \mathrm{C}$. Ammonia, water, carbon dioxide, and some of the unreacted ketones distilled over. The distilled ketone was separated and returned to the reaction mixture. The mixture which gradually became homogenous was maintained at 180$185^{\circ} \mathrm{C}$ for $4-5$ hours. When the reaction was complete, the mixture was cooled and stirred thoroughly with twice its volume of water. The aqueous layer was separated and the formyl derivative of the amine (nonaqueous layer) so obtained was refluxed with $100-150 \mathrm{~mL}$ of concentrated hydrochloric acid for 2-3 hours. After the hydrolysis, the reaction mixture was cooled and extracted with ether to remove any unreacted ketone. The aqueous solution was made strongly alkaline with $30 \%$ sodium hydroxide solution and the separated amine was extracted with ether. The ethereal extract was dried over anhydrous sodium sulphate, and after removal of the solvent, the product distilled under reduced pressure.

2.2.2. General Method for the Synthesis of 2-Chloro-N-(1-(4chlorophenyl)ethyl)acetamide (2a) and 2-Chloro-N-(1-(ptolyl)ethyl)acetamide (2b) from (1a) and (1b), Respectively. An ice-cooled aqueous solution of sodium hydroxide $(50 \mathrm{~mL}$, $10 \%)$ was taken in two different well-corked conical flasks; then, $0.1 \mathrm{~mol}$ of synthesized compounds $\mathbf{1 a}$ and $\mathbf{1 b}$ was added in both the flasks very slowly followed by addition of chloroacetyl chloride $(11.93 \mathrm{~mL}, 0.15 \mathrm{~mol})$ dropwise with constant stirring and shaking. The reaction was strongly shaken until odor of chloroacetyl chloride moved out. The $\mathrm{pH}$ of reaction mixture was kept around 9-10 by the addition of sodium hydroxide solution. Filter off the product and solid amides $\mathbf{2} \mathbf{a}$ and $\mathbf{2} \mathbf{b}$ that formed were washed thoroughly with water, dried, and recrystallized from ethanol.

2.2.3. General Method for the Synthesis of N-(1-(4-Chlorophenyl)ethyl)-2-(substituted phenoxy)acetamide (3a-e) and 2-(Substituted phenoxy)-N-(1-(p-tolyl)ethyl)acetamide $(\mathbf{3} \boldsymbol{f}-\mathbf{j})$ Derivatives from $(\mathbf{2 a})$ and $(\mathbf{2 b})$, Respectively. Phenoxy acetamide derivatives were prepared by reacting (2a-2b) $(0.01 \mathrm{~mol})$ with different substituted phenols $(0.01 \mathrm{~mol})$ in presence of anhydrous potassium carbonate $(0.01 \mathrm{~mol})$ and catalytic amount of potassium iodide in refluxing dry acetone. In some cases unreacted phenol was removed from the final product by treating the substance with $10 \% \mathrm{w} / \mathrm{v}$ sodium carbonate solution in water. The compound was then filtered and washed thoroughly with water and recrystallised from appropriate solvent. The completion of the reaction was monitored by TLC.

(1) N-(1-(4-Chlorophenyl)ethyl)-2-phenoxyacetamide (3a). IR $\left(\mathrm{KBr}, \mathrm{cm}^{-1}\right) .3205,3112,3014,2928,1711,1644,1318,1235 .{ }^{1} \mathrm{H}$ NMR (400 MHz, DMSO-d 6 , $\delta$ ppm): 8.15 (s, 1H, NH), 7.526.90 (m, 4H, H-2', H-3, H-5 $\left.5^{\prime}, \mathrm{H}^{\prime} 6^{\prime}\right), 6.81$ (t, 2H, H-3 $3^{\prime \prime}, \mathrm{H}-$ $\left.5^{\prime \prime}\right)$, 6.20-5-93 (m, 3H, H-2" $\left.2^{\prime \prime} \mathrm{H}-4^{\prime \prime}, \mathrm{H}-6^{\prime \prime}\right), 5.80-5.47(\mathrm{~m}$, $1 \mathrm{H}, \mathrm{CH}-3), 5.21\left(\mathrm{~s}, 2 \mathrm{H}, \mathrm{CH}_{2}-2\right), 1.74\left(\mathrm{~d}, 3 \mathrm{H}, \mathrm{CH}_{3}-4\right) .{ }^{13} \mathrm{C}$ NMR (DMSO- $\left.\mathrm{d}_{6}, \delta \mathrm{ppm}\right): 166.4(\mathrm{C}=\mathrm{O}, \mathrm{NHCO}), 159.8(\mathrm{C}$, $\left.\mathrm{C}-1^{\prime \prime}\right), 141.7\left(\mathrm{C}, \mathrm{C}-1^{\prime}\right), 135.4\left(\mathrm{C}, \mathrm{C}-4^{\prime}\right), 130.1\left(\mathrm{CH}, \mathrm{C}-3^{\prime \prime}, 5^{\prime \prime}\right)$, $125.1\left(\mathrm{CH}, \mathrm{C}-3^{\prime}, 5^{\prime}\right), 124.4\left(\mathrm{CH}, \mathrm{C}-2^{\prime}, 6^{\prime}\right), 120.8\left(\mathrm{CH}, \mathrm{C}-4^{\prime \prime}\right)$, $116.1\left(\mathrm{CH}, \mathrm{C}-2^{\prime \prime}, 6^{\prime \prime}\right), 68.4\left(\mathrm{CH}_{2}, \mathrm{C}-2\right), 55.9(\mathrm{CH}, \mathrm{C}-3), 25.9$ $\left(\mathrm{CH}_{3}, \mathrm{C}-4\right)$. mass: $\mathrm{m} / \mathrm{z} 289\left(\mathrm{M}^{+}\right), 290(\mathrm{M}+1,17.5 \%), 291(\mathrm{M}+2$, $33.4 \%)$. Anal. Calc. For $\mathrm{C}_{16} \mathrm{H}_{16} \mathrm{ClNO}_{2}$ : C 66.32, H 5.57, N 4.83. Found: C 66.28, H 5.53, N 4.85 .

(2) 2-(4-Bromophenoxy)-N-(1-(4-chlorophenyl)ethyl)acetamide (3b). IR (KBr, cm ${ }^{-1}$ ): 3245, 3056, 3017, 2915, 1678, 1622, 1335, 1105. ${ }^{1} \mathrm{H}$ NMR $\left(400 \mathrm{MHz}, \mathrm{DMSO}-\mathrm{d}_{6}, \delta \mathrm{ppm}\right): 8.25(\mathrm{~s}, 1 \mathrm{H}$, $\mathrm{NH})$, 7.81-7.12 (m, 6H, H-2', H-3', H-5', H-6', H-3" , H-5 ${ }^{\prime \prime}$ ), 6.80 (d, 2H, H-2", H-6" $), 6.31-6.24$ (m, 1H, CH-3), 5.34 (s, $\left.2 \mathrm{H}, \mathrm{CH}_{2}-2\right), 1.63$ (d, 3H, $\left.\mathrm{CH}_{3}-4\right) .{ }^{13} \mathrm{C}$ NMR (DMSO-d 6 , $\delta$ ppm): $170.1(\mathrm{C}=\mathrm{O}, \mathrm{NHCO}), 162.5\left(\mathrm{C}, \mathrm{C}-1^{\prime \prime}\right), 142.4$ (C, C$\left.1^{\prime}\right), 139.8\left(\mathrm{C}, \mathrm{C}-4^{\prime}\right), 138.7\left(\mathrm{CH}, \mathrm{C}-3^{\prime \prime}, 5^{\prime \prime}\right), 127.2\left(\mathrm{CH}, \mathrm{C}-3^{\prime}, 5^{\prime}\right)$, $123.8\left(\mathrm{CH}, \mathrm{C}-2^{\prime}, 6^{\prime}\right), 120.8\left(\mathrm{CH}, \mathrm{C}-2^{\prime \prime}, 6^{\prime \prime}\right), 111.9\left(\mathrm{C}, \mathrm{C}-4^{\prime \prime}\right)$, $66.7\left(\mathrm{CH}_{2}, \mathrm{C}-2\right), 50.9(\mathrm{CH}, \mathrm{C}-3), 23.8\left(\mathrm{CH}_{3}, \mathrm{C}-4\right)$. mass: $\mathrm{m} / \mathrm{z}$ $369\left(\mathrm{M}^{+}\right), 367$ (M-2, 76.5\%), $371(\mathrm{M}+2,25.3 \%)$. Anal. Calc. For $\mathrm{C}_{16} \mathrm{H}_{15} \mathrm{BrClNO}_{2}$ : C 52.13, H 4.10, N 3.80. Found: C 52.09, $\mathrm{H} 4.13, \mathrm{~N} 3.77$.

(3) N-(1-(4-Chlorophenyl)ethyl)-2-(4-nitrophenoxy)acetamide (3c). IR (KBr, cm $\left.{ }^{-1}\right)$ : 3255, 3218, 3122, 2817, 1686, 1533, 1528, 1213, 1110. ${ }^{1} \mathrm{H}$ NMR (400 MHz, DMSO-d $6, \delta$ ppm): 8.25 (d, $\left.2 \mathrm{H}, \mathrm{H}-3^{\prime \prime}, \mathrm{H}-5^{\prime \prime}\right), 7.92$ (s, 1H, NH), 7.81-7.35 (m, 4H, H-2', H$\left.3^{\prime}, \mathrm{H}-5^{\prime}, \mathrm{H}-6^{\prime}\right), 7.17$ (d, 2H, H-2", H-6 $\left.{ }^{\prime \prime}\right), 5.78-5.31$ (m, $1 \mathrm{H}$, $\mathrm{CH}-3), 4.92$ (s, 2H, $\left.\mathrm{CH}_{2}-2\right), 2.11\left(\mathrm{~d}, 3 \mathrm{H}, \mathrm{CH}_{3}-4\right) .{ }^{13} \mathrm{C} \mathrm{NMR}$ (DMSO-d ${ }_{6}, \delta$ ppm): $170.5(\mathrm{C}=\mathrm{O}, \mathrm{NHCO}), 163.7\left(\mathrm{C}, \mathrm{C}-1^{\prime \prime}\right)$, $148.4\left(\mathrm{C}, \mathrm{C}-4^{\prime \prime}\right), 145.5\left(\mathrm{C}, \mathrm{C}-1^{\prime}\right), 139.1\left(\mathrm{C}, \mathrm{C}-4^{\prime}\right), 132.4(\mathrm{CH}, \mathrm{C}-$ $\left.3^{\prime}, 5^{\prime}\right), 130.4\left(\mathrm{CH}, \mathrm{C}-2^{\prime}, 6^{\prime}\right), 129.7\left(\mathrm{CH}, \mathrm{C}-3^{\prime \prime}, 5^{\prime \prime}\right), 120.7(\mathrm{CH}$, $\left.\mathrm{C}-2^{\prime \prime}, 6^{\prime \prime}\right), 71.4\left(\mathrm{CH}_{2}, \mathrm{C}-2\right), 69.1(\mathrm{CH}, \mathrm{C}-3), 35.4\left(\mathrm{CH}_{3}, \mathrm{C}-\right.$ 4). mass: $\mathrm{m} / \mathrm{z} 334\left(\mathrm{M}^{+}\right), 335(\mathrm{M}+1,18.7 \%), 336(\mathrm{M}+2,34.1 \%)$. Anal. Cacl. For $\mathrm{C}_{16} \mathrm{H}_{15} \mathrm{ClN}_{2} \mathrm{O}_{4}$ : C 57.41, $\mathrm{H}$ 4.52, N 8.37. Found: C 57.45, H 4.48, N 8.32. 
(4) 2-(4-(Tert-butyl)phenoxy)-N-(1-(4-chlorophenyl)ethyl)acetamide (3d). IR (KBr, cm $\left.{ }^{-1}\right)$ : 3217, 3181, 2973, 2810, 1688, 1611, 1290, 1176. ${ }^{1} \mathrm{H}$ NMR (400 MHz, DMSO-d 6 , $\delta$ ppm): $8.22(\mathrm{~s}$, $1 \mathrm{H}, \mathrm{NH}), 8.10-7.37$ (m, 6H, H-2' $, \mathrm{H}-3^{\prime}, \mathrm{H}-5^{\prime}, \mathrm{H}-6^{\prime}, \mathrm{H}-3^{\prime \prime}, \mathrm{H}-$ $5^{\prime \prime}$ ), 7.21 (d, 2H, H-2" $2^{\prime \prime}$ H-6 $\left.{ }^{\prime \prime}\right), 5.20-5.13$ (m, 1H, CH-3), 4.94 (s, $\left.2 \mathrm{H}, \mathrm{CH}_{2}-2\right), 3.11\left(\mathrm{~d}, 3 \mathrm{H}, \mathrm{CH}_{3}-4\right), 2.83\left(\mathrm{~s}, 9 \mathrm{H},\left(\mathrm{CH}_{3}\right)_{3}\right) .{ }^{13} \mathrm{C}$ NMR (DMSO-d ${ }_{6}, \delta$ ppm): $169.8(\mathrm{C}=\mathrm{O}, \mathrm{NHCO}), 159.4$ (C, C$\left.1^{\prime \prime}\right), 151.8\left(\mathrm{C}, \mathrm{C}-4^{\prime \prime}\right), 137.8\left(\mathrm{C}, \mathrm{C}-1^{\prime}\right), 131.8\left(\mathrm{C}, \mathrm{C}-4^{\prime}\right), 130.4(\mathrm{CH}$, C-3', $\left.5^{\prime}\right), 128.1\left(\mathrm{CH}, \mathrm{C}-2^{\prime}, 6^{\prime}\right), 123.4\left(\mathrm{CH}, \mathrm{C}-3^{\prime \prime}, 5^{\prime \prime}\right), 110.4$ $\left(\mathrm{CH}, \mathrm{C}-2^{\prime \prime}, 6^{\prime \prime}\right), 64.5\left(\mathrm{CH}_{2}, \mathrm{C}-2\right), 61.8(\mathrm{CH}, \mathrm{C}-3), 41.4(\mathrm{C}$, C- $\left.\left(\mathrm{CH}_{3}\right)_{3}\right), 40.9\left(\left(\mathrm{CH}_{3}\right)_{3}\right), 19.7\left(\mathrm{CH}_{3}, \mathrm{C}-4\right)$. mass: $\mathrm{m} / \mathrm{z} 345$ $\left(\mathrm{M}^{+}\right), 346(\mathrm{M}+1,22.3 \%), 347(\mathrm{M}+2,32.4 \%)$. Anal. Calc. For $\mathrm{C}_{20} \mathrm{H}_{24} \mathrm{ClNO}_{2}$ : C 69.45, H 6.99, N 4.05. Found: C 69.41, H 6.94, N 4.10 .

(5) N-(1-(4-Chlorophenyl)ethyl)-2-(4-methoxyphenoxy)acetamid $e(3 \mathrm{e})$. IR (KBr, cm $\left.{ }^{-1}\right): 3216,3122,2988,2917,2713,1677,1609$, 1200, 1174. ${ }^{1} \mathrm{H}$ NMR (400 MHz, DMSO-d 6 , $\delta$ ppm): 8.67 (s, $1 \mathrm{H}, \mathrm{NH}$ ), 7.94-7.36 (m, 4H, H-2', H-3', H-5', H-6 ${ }^{\prime}$ ), 7.20 (s, $\left.4 \mathrm{H}, \mathrm{H}-2^{\prime \prime}, \mathrm{H}-3^{\prime \prime}, \mathrm{H}-5^{\prime \prime}, \mathrm{H}-6^{\prime \prime}\right), 5.43-5.21$ (m, 1H, CH-3), 5.11 (s, $\left.2 \mathrm{H}, \mathrm{CH}_{2}-2\right), 4.64$ (s, 3H, $\left.\mathrm{OCH}_{3}\right), 2.36$ (d, 3H, $\left.\mathrm{CH}_{3}-4\right)$. ${ }^{13} \mathrm{C} \mathrm{NMR}\left(\mathrm{DMSO}-\mathrm{d}_{6}, \delta \mathrm{ppm}\right): 172.9(\mathrm{C}=\mathrm{O}, \mathrm{NHCO}), 154.2(\mathrm{C}$, $\left.\mathrm{C}-4^{\prime \prime}\right), 152.8\left(\mathrm{C}, \mathrm{C}-1^{\prime \prime}\right), 139.8\left(\mathrm{C}, \mathrm{C}-1^{\prime}\right), 137.2\left(\mathrm{C}, \mathrm{C}-4^{\prime}\right), 131.2$ $\left(\mathrm{CH}, \mathrm{C}-3^{\prime}, 5^{\prime}\right), 130.1\left(\mathrm{CH}, \mathrm{C}-2^{\prime}, 6^{\prime}\right), 121.1\left(\mathrm{CH}, 2^{\prime \prime}, 3^{\prime \prime}, 5^{\prime \prime}\right.$, $\left.6^{\prime \prime}\right), 73.3\left(\mathrm{CH}_{2}, \mathrm{C}-2\right), 61.4\left(\mathrm{CH}_{3}, \mathrm{OCH}_{3}\right), 60.2(\mathrm{CH}, \mathrm{C}-3), 30.1$ $\left(\mathrm{CH}_{3}, \mathrm{C}-4\right)$. mass: $\mathrm{m} / \mathrm{z} 319\left(\mathrm{M}^{+}\right), 320(\mathrm{M}+1,19.6 \%), 321(\mathrm{M}+2$, 34.1\%). Anal. Calc. For: $\mathrm{C}_{17} \mathrm{H}_{18} \mathrm{ClNO}_{3}$ : C 63.85, H 5.67, N 4.38. Found: C 63.81, H 5.70, N 4.32.

(6) 2-Phenoxy-N-(1-(p-tolyl)ethyl)acetamide (3f). IR (KBr, $\left.\mathrm{cm}^{-1}\right): 3266,3033,2895,1654,1622,1278,1112 .{ }^{1} \mathrm{H}$ NMR $\left(400 \mathrm{MHz}, \mathrm{DMSO}-\mathrm{d}_{6}, \delta \mathrm{ppm}\right): 8.32(\mathrm{~s}, 1 \mathrm{H}, \mathrm{NH}), 8.11(\mathrm{t}, 2 \mathrm{H}$, $\left.\mathrm{H}-3^{\prime \prime}, \mathrm{H}-5^{\prime \prime}\right), 7.46-7.31$ (m, 4H, H-2', H-3', H-5', H-6 $), 7.12-$ $6.93\left(\mathrm{~m}, 3 \mathrm{H}, \mathrm{H}-2^{\prime \prime}, \mathrm{H}-4^{\prime \prime}, \mathrm{H}-6^{\prime \prime}\right), 6.81-6.78$ (m, 1H, CH-3), 6.61 (s, $\left.2 \mathrm{H}, \mathrm{CH}_{2}-2\right), 3.36\left(\mathrm{~s}, 3 \mathrm{H}, \mathrm{CH}_{3}-4^{\prime}\right), 2.45\left(\mathrm{~d}, 3 \mathrm{H}, \mathrm{CH}_{3}-4\right) .{ }^{13} \mathrm{C}$ NMR (DMSO-d 6 , $\delta$ ppm): $171.8(\mathrm{C}=\mathrm{O}, \mathrm{NHCO}), 167.4$ (C, C$\left.1^{\prime \prime}\right), 151.2\left(\mathrm{C}, \mathrm{C}-1^{\prime}\right), 150.1\left(\mathrm{C}, \mathrm{C}-4^{\prime}\right), 132.4\left(\mathrm{CH}, \mathrm{C}-3^{\prime \prime}, 5^{\prime \prime}\right), 130.6$ $\left(\mathrm{CH}, \mathrm{C}-3^{\prime}, 5^{\prime}\right), 129.8\left(\mathrm{CH}, \mathrm{C}-2^{\prime}, 6^{\prime}\right), 127.1\left(\mathrm{CH}, \mathrm{C}-4^{\prime \prime}\right), 122.7$ $\left(\mathrm{CH}, \mathrm{C}-2^{\prime \prime}, 6^{\prime \prime}\right), 66.8\left(\mathrm{CH}_{2}, \mathrm{C}-2\right), 57.1(\mathrm{CH}, \mathrm{C}-3), 20.9\left(\mathrm{CH}_{3}\right.$, C-4), $18.7\left(\mathrm{CH}_{3}, \mathrm{CH}_{3}-\mathrm{C}^{\prime}\right)$. mass: $\mathrm{m} / \mathrm{z} 269\left(\mathrm{M}^{+}\right), 270(\mathrm{M}+1$, 19.6\%), $271(\mathrm{M}+2,2.7 \%)$. Anal. Calc. For $\mathrm{C}_{17} \mathrm{H}_{19} \mathrm{NO}_{2}$ : C 75.81, H 7.11, N 5.20. Found: C 75.84, H 7.05, N 5.24.

(7) 2-(4-Bromophenoxy)-N-(1-(p-tolyl)ethyl)acetamide (3g). IR $\left(\mathrm{KBr}, \mathrm{cm}^{-1}\right): 3256,3078,2895,1674,1556,1279,1090 .{ }^{1} \mathrm{H}$ NMR (400 MHz, DMSO-d 6 , $\delta$ ppm): 8.45 (s, 1H, NH), 8.21 (d, 2H, H-3 $\left.3^{\prime \prime}, \mathrm{H}-5^{\prime \prime}\right), 7.81-7.73$ (m, 4H, H-2 ${ }^{\prime}, \mathrm{H}-3^{\prime}, \mathrm{H}-5^{\prime}, \mathrm{H}-$ $6^{\prime}$ ), 7.47 (d, 2H, H-2" ${ }^{\prime \prime}$ H-6" ${ }^{\prime \prime}$, 5.61-5.53 (m, 1H, CH-3), 5.44 (s, $\left.2 \mathrm{H}, \mathrm{CH}_{2}-2\right), 4.13\left(\mathrm{~s}, 3 \mathrm{H}, \mathrm{CH}_{3}-4^{\prime}\right), 2.78\left(\mathrm{~d}, 3 \mathrm{H}, \mathrm{CH}_{3}-4\right) .{ }^{13} \mathrm{C}$ NMR (DMSO-d ${ }_{6}, \delta$ ppm): $169.2(\mathrm{C}=\mathrm{O}, \mathrm{NHCO}), 161.8$ (C, C$\left.1^{\prime \prime}\right), 142.9\left(\mathrm{C}, \mathrm{C}-1^{\prime}\right), 141.4\left(\mathrm{C}, \mathrm{C}-4^{\prime}\right), 139.9\left(\mathrm{CH}, \mathrm{C}-3^{\prime \prime}, 5^{\prime \prime}\right), 126.4$ $\left(\mathrm{CH}, \mathrm{C}-3^{\prime}, 5^{\prime}\right), 123.8\left(\mathrm{CH}, \mathrm{C}-2^{\prime}, 6^{\prime}\right), 116.2\left(\mathrm{CH}, \mathrm{C}-1^{\prime \prime}, 6^{\prime \prime}\right), 114.8$ $\left(\mathrm{C}, \mathrm{C}-4^{\prime \prime}\right), 68.6\left(\mathrm{CH}_{2}, \mathrm{C}-2\right), 51.3(\mathrm{CH}, \mathrm{C}-3), 26.7\left(\mathrm{CH}_{3}, \mathrm{C}-4\right)$, $25.8\left(\mathrm{CH}_{3}, \mathrm{CH}_{3}-\mathrm{C}^{\prime}\right)$. mass: $\mathrm{m} / \mathrm{z} 347\left(\mathrm{M}^{+}\right), 348(\mathrm{M}+1,18.2 \%)$, $349\left(\mathrm{M}+2\right.$, 99.8\%), Anal. Calc. For $\mathrm{C}_{17} \mathrm{H}_{18} \mathrm{BrNO}_{2}$ : C 58.63, $\mathrm{H}$ 5.21, N 4.02. Found: C 58.69, H 5.17, N 4.08.

(8) 2-(4-Nitrophenoxy)-N-(1-(p-tolyl)ethyl)acetamide (3h). IR $\left(\mathrm{KBr}, \mathrm{cm}^{-1}\right):$ 3219, 3078, 2917, 1678, 1596, 1534, 1267, 1034.
${ }^{1} \mathrm{H}$ NMR (400 MHz, DMSO-d 6 , $\delta$ ppm): 8.37 (d, 2H, H-3", H-5 ${ }^{\prime \prime}$ ), 8.13 (s, 1H, NH), 7.92 (d, 2H, H-2" , H-6 $\left.{ }^{\prime \prime}\right), 7.79-7.53$ (m, $\left.4 \mathrm{H}, \mathrm{H}-2^{\prime}, \mathrm{H}-3^{\prime}, \mathrm{H}-5^{\prime}, \mathrm{H}-6^{\prime}\right), 6.78-6.57(\mathrm{~m}, 1 \mathrm{H}, \mathrm{CH}-3), 5.30$ (s, $\left.2 \mathrm{H}, \mathrm{CH}_{2}-2\right), 3.39$ (s, 3H, $\left.\mathrm{CH}_{3}-4^{\prime}\right), 2.84\left(\mathrm{~d}, 3 \mathrm{H}, \mathrm{CH}_{3}-4\right) .{ }^{13} \mathrm{C}$ NMR (DMSO-d ${ }_{6}, \delta$ ppm): $168.4(\mathrm{C}=\mathrm{O}, \mathrm{NHCO}), 167.1$ (C, C$\left.1^{\prime \prime}\right), 161.9\left(\mathrm{C}, \mathrm{C}-4^{\prime \prime}\right), 157.8\left(\mathrm{C}, \mathrm{C}-1^{\prime}\right), 139.6\left(\mathrm{C}, \mathrm{C}-4^{\prime}\right), 131.6(\mathrm{CH}$, $\left.\mathrm{C}-3^{\prime}, 5^{\prime}\right), 129.1\left(\mathrm{CH}, \mathrm{C}-3^{\prime \prime}, 5^{\prime \prime}\right), 127.4\left(\mathrm{CH}, \mathrm{C}-2^{\prime}, 6^{\prime}\right), 120.4(\mathrm{CH}$, $\left.\mathrm{C}-2^{\prime \prime}, 6^{\prime \prime}\right), 71.8\left(\mathrm{CH}_{2}, \mathrm{C}-2\right), 68.4(\mathrm{CH}, \mathrm{C}-3), 44.6\left(\mathrm{CH}_{3}, \mathrm{C}-4\right)$, $41.9\left(\mathrm{CH}_{3}, \mathrm{CH}_{3}-\mathrm{C}^{\prime}\right)$. mass: $\mathrm{m} / \mathrm{z} 314\left(\mathrm{M}^{+}\right), 315(\mathrm{M}+1,19.1 \%)$, $316(\mathrm{M}+2,2.9 \%)$. Anal. Calc. For $\mathrm{C}_{17} \mathrm{H}_{18} \mathrm{~N}_{2} \mathrm{O}_{4}$ : C 64.96, $\mathrm{H}$ 5.77, N 8.91. Found: C 64.91, H 5.78, N 8.97.

(9) 2-(4-(Tert-butyl)phenoxy)-N-(1-(p-tolyl)ethyl)acetamide (3i). IR $\left(\mathrm{KBr}, \mathrm{cm}^{-1}\right)$ : 3243, 3056, 2978, 2943, 1635, 1575, $1266,1044 .{ }^{1} \mathrm{H}$ NMR $\left(400 \mathrm{MHz}, \mathrm{DMSO}-\mathrm{d}_{6}, \delta \mathrm{ppm}\right): 8.51(\mathrm{~s}$, $1 \mathrm{H}, \mathrm{NH}), 8.33$ (d, 2H, H-3", H-5") $8.27-8.11$ (m, 4H, H-2', H-3', H-5', H-6'), 7.53 (d, 2H, H-2 $\left.{ }^{\prime \prime}, \mathrm{H}-6^{\prime \prime}\right), 5.11-4.28$ (m, $1 \mathrm{H}, \mathrm{CH}-3), 4.37$ (s, $\left.2 \mathrm{H}, \mathrm{CH}_{2}-2\right), 3.81$ (s, $\left.3 \mathrm{H}, \mathrm{CH}_{3}-4^{\prime}\right), 3.16$ $\left(\mathrm{d}, 3 \mathrm{H}, \mathrm{CH}_{3}-4\right), 2.76\left(\mathrm{~s}, 9 \mathrm{H},\left(\mathrm{CH}_{3}\right)_{3}\right) .{ }^{13} \mathrm{C} \mathrm{NMR}\left(\mathrm{DMSO}-\mathrm{d}_{6}\right.$, $\delta \mathrm{ppm}): 170.8(\mathrm{C}=\mathrm{O}, \mathrm{NHCO}), 158.4\left(\mathrm{C}, \mathrm{C}-1^{\prime \prime}\right), 151.3(\mathrm{C}$, $\left.\mathrm{C}-4^{\prime \prime}\right), 139.1\left(\mathrm{C}, \mathrm{C}-1^{\prime}\right), 138.2\left(\mathrm{C}, \mathrm{C}-4^{\prime}\right), 136.4\left(\mathrm{CH}, \mathrm{C}-3^{\prime}, 5^{\prime}\right)$, $134.1\left(\mathrm{CH}, \mathrm{C}-3^{\prime \prime}, 5^{\prime \prime}\right), 130.8\left(\mathrm{CH}, \mathrm{C}-2^{\prime}, 6^{\prime}\right), 122.6\left(\mathrm{CH}, \mathrm{C}-2^{\prime \prime}\right.$, $\left.6^{\prime \prime}\right), 74.6\left(\mathrm{CH}_{2}, \mathrm{C}-2\right), 71.4(\mathrm{CH}, \mathrm{C}-3), 51.7\left(\mathrm{C}, \mathrm{C}-\left(\mathrm{CH}_{3}\right)_{3}\right), 46.2$ $\left(\left(\mathrm{CH}_{3}\right)_{3}\right), 38.1\left(\mathrm{CH}_{3}, \mathrm{C}-4\right), 36.7\left(\mathrm{CH}_{3}, \mathrm{CH}_{3}-\mathrm{C}^{\prime}\right)$. mass: $\mathrm{m} / \mathrm{z}$ $325\left(\mathrm{M}^{+}\right), 326(\mathrm{M}+1,23.1 \%), 327(\mathrm{M}+2,3.6 \%)$. Anal. Calc. For $\mathrm{C}_{21} \mathrm{H}_{27} \mathrm{NO}_{2}$ : C 77.50, $\mathrm{H} 8.36, \mathrm{~N} 4.30$. Found: $\mathrm{C} 77.43, \mathrm{H}$ 8.40, N 4.36 .

(10) 2-(4-Methoxyphenoxy)-N-(1-(p-tolyl)ethyl)acetamide (3j). IR $\left(\mathrm{KBr}, \mathrm{cm}^{-1}\right)$ : 3233, 2935, 2907, 2811, 1644, 1576, 1308, 1017. ${ }^{1} \mathrm{H}$ NMR (400 MHz, DMSO-d 6 , $\delta$ ppm): 8.44 (s, 1H, NH), 8.21-8.13 (m, 4H, H-2', H-3', H-5', H-6 ${ }^{\prime}$ ), 7.69 (s, 4H, H-2' $\left.\mathrm{H}-3^{\prime \prime}, \mathrm{H}-5^{\prime \prime}, \mathrm{H}-6^{\prime \prime}\right), 5.75-5.61$ (m, $\left.1 \mathrm{H}, \mathrm{CH}-3\right), 5.32$ (s, 2H, $\left.\mathrm{CH}_{2}-2\right), 4.23\left(\mathrm{~s}, 3 \mathrm{H}, \mathrm{OCH}_{3}\right), 2.78\left(\mathrm{~s}, 3 \mathrm{H}, \mathrm{CH}_{3}-4^{\prime}\right), 2.34(\mathrm{~d}, 3 \mathrm{H}$, $\left.\mathrm{CH}_{3}-4\right) .{ }^{13} \mathrm{C}$ NMR (DMSO-d 6 , $\delta$ ppm): 171.2 (C=O, NHCO), $168.8\left(\mathrm{C}, \mathrm{C}-4^{\prime \prime}\right), 161.4\left(\mathrm{C}, \mathrm{C}-1^{\prime \prime}\right), 159.8\left(\mathrm{C}, \mathrm{C}-1^{\prime}\right), 158.4(\mathrm{C}, \mathrm{C}-$ $\left.4^{\prime}\right), 161.4\left(\mathrm{C}, \mathrm{C}-1^{\prime \prime}\right), 157.1\left(\mathrm{CH}, \mathrm{C}-3^{\prime}, 5^{\prime}\right), 152.3\left(\mathrm{CH}, \mathrm{C}-2^{\prime}, 6^{\prime}\right)$, $148.2\left(\mathrm{CH}, \mathrm{C}-2^{\prime \prime}, 3^{\prime \prime}, 5^{\prime \prime}, 6^{\prime \prime}\right), 76.1\left(\mathrm{CH}_{2}, \mathrm{C}-2\right), 71.7\left(\mathrm{CH}_{3}\right.$, $\left.\mathrm{OCH}_{3}\right), 65.4(\mathrm{CH}, \mathrm{C}-3), 32.8\left(\mathrm{CH}_{3}, \mathrm{C}-4\right), 31.7\left(\mathrm{CH}_{3}, \mathrm{CH}_{3}-\right.$ $\left.\mathrm{C4}^{\prime}\right)$. mass: $\mathrm{m} / \mathrm{z} 299\left(\mathrm{M}^{+}\right), 300(\mathrm{M}+1,19.2 \%), 301(\mathrm{M}+2,2.8 \%)$. Anal. Calc. For $\mathrm{C}_{18} \mathrm{H}_{21} \mathrm{NO}_{3}$ : C 72.22, $\mathrm{H}$ 7.07, $\mathrm{N}$ 4.68. Found: C 72.29, H 7.01, N 4.62.

\subsection{Pharmacological Screening}

2.3.1. Animals. Wister albino rats of either gender weighing 140-180 g were obtained. The animals were divided into several groups of five animals each. All the animals were housed under standard ambient environment of temperature $\left(25 \pm 2^{\circ} \mathrm{C}\right)$ and relative humidity of $50 \pm 5 \%$. A $12: 12$ hour light: dark cycle was maintained. All the animals were allowed to have free access to water and standard palletized laboratory animal diet 12 hours prior to pharmacological studies. All the experimental procedures and protocols used in this study were reviewed and approved by the Institutional Animal Ethical Committee (IAEC).

2.3.2. Preparation of Test Compounds. Test samples and the reference drugs were prepared as a suspension in $1 \%$ tween 80 . 
TABLE 1: Characterization data of N-(1-(4-chlorophenyl)ethyl)-2-(Substituted phenoxy)acetamide derivatives (3a-e) and 2-(substituted phenoxy)-N-(1-(p-tolyl)ethyl)acetamide derivatives $(\mathbf{3} \mathbf{f}-\mathbf{j})$.

\begin{tabular}{|c|c|c|c|c|c|}
\hline Compound & $\mathrm{R}$ & Yield (\%) & Melting point ${ }^{*}\left({ }^{\circ} \mathrm{C}\right)$ & Rf value $^{\#}$ & Molecular formula \\
\hline $3 a$ & $\mathrm{H}$ & 61.4 & $171-173$ & 0.37 & $\mathrm{C}_{16} \mathrm{H}_{16} \mathrm{ClNO}_{2}$ \\
\hline $3 b$ & $4-\mathrm{Br}$ & 58.3 & $154-156$ & 0.45 & $\mathrm{C}_{16} \mathrm{H}_{15} \mathrm{BrClNO}_{2}$ \\
\hline $3 c$ & $4-\mathrm{NO}_{2}$ & 59.1 & $165-167$ & 0.41 & $\mathrm{C}_{16} \mathrm{H}_{15} \mathrm{ClN}_{2} \mathrm{O}_{4}$ \\
\hline $3 d$ & $4-\mathrm{C}-\left(\mathrm{CH}_{3}\right)_{3}$ & 62.7 & $205-207$ & 0.50 & $\mathrm{C}_{20} \mathrm{H}_{24} \mathrm{ClNO}_{2}$ \\
\hline $3 e$ & $4-\mathrm{OCH}_{3}$ & 71.0 & $126-128$ & 0.38 & $\mathrm{C}_{17} \mathrm{H}_{18} \mathrm{ClNO}_{3}$ \\
\hline $3 f$ & $\mathrm{H}$ & 67.8 & $154-156$ & 0.32 & $\mathrm{C}_{17} \mathrm{H}_{19} \mathrm{NO}_{2}$ \\
\hline $3 g$ & $4-\mathrm{Br}$ & 57.2 & $189-191$ & 0.46 & $\mathrm{C}_{17} \mathrm{H}_{18} \mathrm{BrNO}_{2}$ \\
\hline $3 h$ & $4-\mathrm{NO}_{2}$ & 74.5 & $148-150$ & 0.51 & $\mathrm{C}_{17} \mathrm{H}_{18} \mathrm{~N}_{2} \mathrm{O}_{4}$ \\
\hline $3 \mathbf{i}$ & $4-\mathrm{C}-\left(\mathrm{CH}_{3}\right)_{3}$ & 65.9 & $172-174$ & 0.39 & $\mathrm{C}_{21} \mathrm{H}_{27} \mathrm{NO}_{2}$ \\
\hline $3 \mathbf{j}$ & $4-\mathrm{OCH}_{3}$ & 70.4 & $142-144$ & 0.47 & $\mathrm{C}_{18} \mathrm{H}_{21} \mathrm{NO}_{3}$ \\
\hline
\end{tabular}

${ }^{*}$ Recrystallization with ethanol. ${ }^{\#}$ Stationary phase: silica gel; mobile phase: $\mathrm{n}$-hexane : ethyl acetate $(1: 1)$; iodine vapors as visualizing agent.

Control group received $0.1 \mathrm{~mL}$ of tween 80 suspension orally. The second group (reference) received a dose of $50 \mathrm{mg} / \mathrm{kg}$ suspension of diclofenac sodium. Test groups were treated with a dose of $100 \mathrm{mg} / \mathrm{kg}$ of final synthesized compounds.

2.3.3. Acute Toxicity. The acute toxicity study was carried out according to OECD guidelines [25] to found the successful dose of the test compounds after getting ethical clearance. Wister albino rats of either sex weighing between 140 and $180 \mathrm{~g}$ were divided into several groups of 5 animals each. Animals were starved for 12 hours prior to test. On the day of the experiment, animals were treated with different compounds to different groups in an increasing order of 10 , $20,100,200$, and $1000 \mathrm{mg} / \mathrm{kg}$ body weight orally. The animals were then observed continuously for 3 hours for common behavioral and autonomic profiles and then every $30 \mathrm{~min}$ for next 4 hours and finally for the next 24 hours or till death.

As per above toxicity test, it was observed that at a highest dose of $1000 \mathrm{mg} / \mathrm{kg}$ body weight, animals were found to be safe. But few changes were found in the behavioral reaction like touch response, alertness, and restlessness. Therefore, $1 / 10$ th of the highest tolerated dose, that is, $100 \mathrm{mg} / \mathrm{kg}$ body weight (b.w.), was chosen for the studies.

2.3.4. Anticancer Activity. Compounds $\mathbf{3 a}-\mathbf{j}$ were biologically evaluated for in vitro cytotoxicity using sulforhodamine B assay $(\mathrm{SRB})$ based cellular protein content determination against two human cancer cell lines that contained MCF-7 (breast) and SK-N-SH (Neuroblastoma), respectively. Adriamycin drug was taken as positive control and the results are reported as percent control growth. In this current protocol, each cell line is preincubated on microtitre plate. Results for each test compound are reported as the percent growth of treated cells is compared with the untreated control cells. The compounds which reduce growth of cell lines to $32 \%$ or less are considered active.

2.3.5. Anti-Inflammatory Activity. Carrageenan induced rat paw edema method [26] was employed for screening of the anti-inflammatory activity of the synthesized compounds listed in Table 1. The animals were divided into twelve groups of five each. One hour after oral administration of the drug, acute inflammation was produced by preparing aqueous suspension of carrageenan $(1 \% \mathrm{w} / \mathrm{v}, 0.1 \mathrm{~mL})$ which was injected in the right hind paw in the subplanter region of each rat. A mark was applied on the leg at the malleolus to facilitate subsequent readings. The paw volume was measured plethysmometrically at $30 \mathrm{~min}, 2 \mathrm{hr}$, and $4 \mathrm{hr}$ after the injection of carrageenan. The $\%$ inhibition was calculated by applying the Newbould formula [27]:

$$
\text { \%Inhibition }=\left(1-\frac{V_{t}}{V_{c}}\right) \times 100,
$$

where $V_{t}$ and $V_{c}$ are the mean change in paw volume of treated and control rats, respectively.

2.3.6. Analgesic Activity. The compounds exhibited an important analgesic activity as per Eddy's hot plate method [28]. Animals were individually placed on a hot plate maintained at a constant temperature $\left(55^{\circ} \mathrm{C}\right)$ and the reaction of animals such as paw licking or jump response (whichever appears first) was taken as the end point. A cut-off time of 15 seconds was taken as maximum analgesic response to avoid any injury of the paws. The reference group was administered with a dose of $50 \mathrm{mg} / \mathrm{kg}$ of the suspension of diclofenac sodium (standard). The reaction time for each animal was noted on the hot plate at 30,60, and 90 minutes after drug administration.

2.3.7. Statistical Analysis. The results of anticancer, antiinflammatory, and analgesic activities are shown in the Tables 2,3 , and 4 , respectively. The results were expressed as mean \pm SEM and were analyzed using one-way analysis of variance (ANOVA) followed by Dunnett's $t$-test. The probability of 0.05 or less was considered statistically significant. Statistical analysis was computed with the GraphPad Prism software version 5.01, GraphPad Software Inc. USA.

\section{Results and Discussion}

A series of titled derivatives N-(1-(4-chlorophenyl)ethyl)-2(substituted phenoxy)acetamide 3 (a-e) derivatives and 2(substituted phenoxy)-N-(1-(p-tolyl)ethyl)acetamide $\mathbf{3}(\mathbf{f}-\mathbf{j})$ 

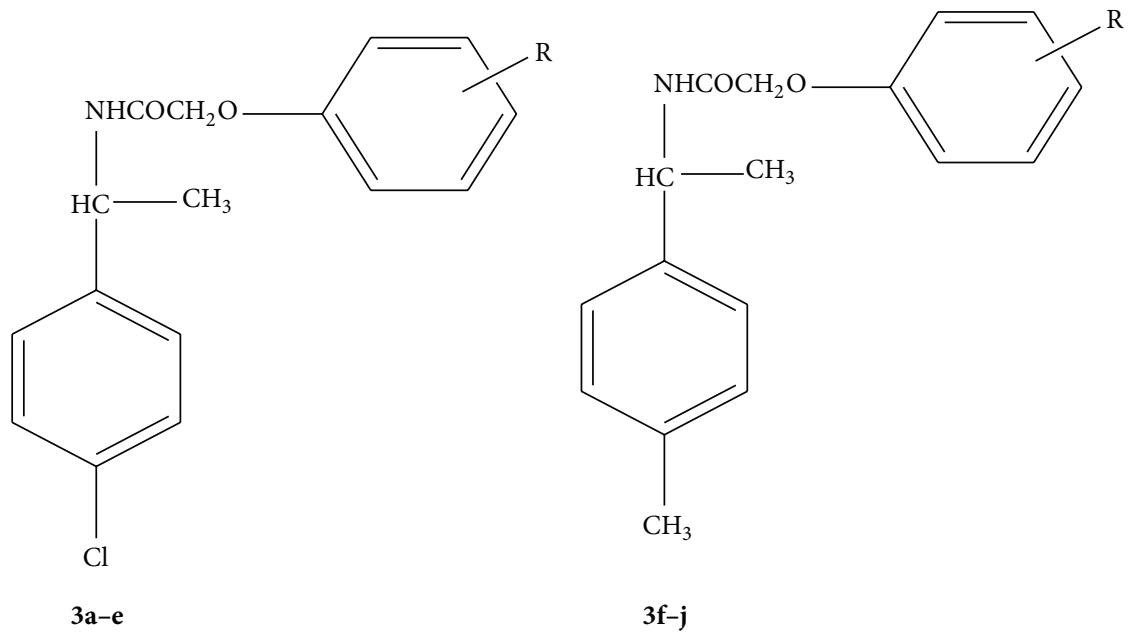

FIGURE 2: General structure of the synthesized compounds.

TABLE 2: Result of anticancer activity.

\begin{tabular}{lccc}
\hline Compound & \multicolumn{2}{c}{ Control growth } & \\
& $\begin{array}{c}\text { MCF-7 } \\
\text { (breast) }\end{array}$ & $\begin{array}{c}\text { SK-N-SH } \\
\text { (neuroblastoma) }\end{array}$ & Activity \\
\hline 3a & 88.9 & 88.2 & Inactive \\
3b $^{*}$ & 30.5 & 67.6 & Active \\
3c** $^{* *}$ & 31.6 & 32.3 & Active \\
$\mathbf{3 d}$ & 54.0 & 72.1 & Inactive \\
$\mathbf{3 e}$ & 88.0 & 95.7 & Inactive \\
$\mathbf{3} \mathbf{f}^{* *}$ & 71.9 & 32.1 & Active \\
$\mathbf{3 g}$ & 44.4 & 69.1 & Inactive \\
$\mathbf{3 h}$ & 70.0 & 71.8 & Inactive \\
$\mathbf{3 i}$ & 58.3 & 73.1 & Inactive \\
$\mathbf{3 j}$ & 74.0 & 80.1 & Inactive \\
\hline
\end{tabular}

${ }^{*}$ Active against MCF-7 (breast) cell line.

${ }^{* *}$ Active against SK-N-SH (neuroblastoma) cell line.

${ }^{* * *}$ Active against both MCF-7 (breast) and SK-N-SH (neuroblastoma) cell lines.

Growth percentages less than 32 are considered as active.

derivatives (Figures 2 and 3) was synthesized as per scheme. 1(4-chlorophenyl)ethanone and 1-(p-tolyl)ethanone were separately treated with ammonium carbonate and formic acid resulting in the formation of amines $\mathbf{1 a}$ and $\mathbf{1 b}$, respectively. These amines on chloroacetylation with chloroacetyl chloride at $0^{\circ} \mathrm{C}$ in $10 \%$ sodium hydroxide medium give chloro compounds $\mathbf{2} \mathbf{a}$ and $\mathbf{2} \mathbf{b}$ which converted to $(\mathbf{3} \mathbf{a}-\mathbf{j})$ by the reaction with different substituted phenols in presence of potassium iodide and potassium carbonate in dry acetone as a solvent. The structure of newly synthesized compounds (Figures 2 and 3) was confirmed by spectral data (IR, ${ }^{1} \mathrm{H}$ NMR, ${ }^{13} \mathrm{C}-\mathrm{NMR}$ and mass); Table 1 shows the physical data of compounds $(\mathbf{3} \mathbf{a}-\mathbf{j})$.

Further IR spectrum of compounds $(\mathbf{3} \mathbf{a}-\mathbf{j})$ showed characteristic absorption bands at range of $3205-3266 \mathrm{~cm}^{-1}$ were attributed to $\mathrm{NH}, 1635-1711 \mathrm{~cm}^{-1}$ accounting for $\mathrm{C}=\mathrm{O}$ of amide group and $1533-1644$ for $\mathrm{C}=\mathrm{C}$ in the aromatic ring. Two peaks at range of 1200-1335 and range of 1017-1235 indicate the presence of $\mathrm{C}-\mathrm{O}-\mathrm{C}$ linkage. The structure of compounds was further supported by mass spectral data.

All compounds $\mathbf{3} \mathbf{a}-\mathbf{j}$ were subjected for preliminary toxicity test as per organization for Economic Co-operation and Development (OECD) guidelines in rats and $100 \mathrm{mg} / \mathrm{kg}$ was used as therapeutic dose. Acute anti-inflammatory activity was performed by carrageenan induced rat paw edema method by Newbould [26]. Diclofenac sodium was used as a reference standard. Compounds $\mathbf{3 b}, \mathbf{3 c}$, and $\mathbf{3 g}$ exhibited potent anti-inflammatory activity similar to the standard (Table 3, Figure 4). The analgesic effects of compounds $3 \mathbf{b}, 3 \mathbf{c}$, $3 \mathrm{e}, \mathbf{3 g}$, and $\mathbf{3 h}$ were found to be nearly of standard (Table 4 , Figure 5).

Compounds $3 \mathrm{c}$ were found to possess anticancer, antiinflammatory, and analgesic activities nearly to the standard because of the presence of $\mathrm{NO}_{2}$ group and $\mathrm{Br}$ groups at position 4 of the phenoxy ring.

From the comprehensive analysis of the results in current studies, we conclude that synthesized compounds have anticancer, anti-inflammatory, and analgesic activities because of the presence of 1-phenylethylamine as basic ring. In analysis of these observations, we conclude that this series $(\mathbf{3} \mathbf{a}-\mathbf{j})$ could be developed and explored as a novel class of anticancer and NSAIDs. However, further detailed pharmacological program is required to recognize the potent molecule without various side effects.

3.1. Structure Activity Relationship (SAR). By comparing the cytotoxic potency of newly synthesized compounds $\mathbf{3} \mathbf{a}-\mathbf{j}$, a few inferences could be drawn as follows. (i) The presence of tert-butyl, methoxy, and nitro substituent at 4 positions does not contribute towards cytotoxicity as can be seen in compounds $\mathbf{3 d}, \mathbf{3} \mathbf{j}$, and $\mathbf{3 h}$ which showed poor growth inhibition against both of the cancer cell lines used. (ii) The presence of $\mathrm{Br}$ group at 4 positions contributes significantly towards the enhancement of inhibitory activity. (iii) The presence of $\mathrm{NO}_{2}$ group at 4 positions in the phenoxy nucleus attached to 
TABLE 3: Result of anti-inflammatory activity.

\begin{tabular}{|c|c|c|c|c|c|c|}
\hline \multirow{2}{*}{ Compound } & \multicolumn{3}{|c|}{ Mean changes in paw edema $(\mathrm{mL})$ mean \pm SEM } & \multicolumn{3}{|c|}{$\%$ Inhibition } \\
\hline & $30 \mathrm{~min}$ & $2 \mathrm{hr}$ & $4 \mathrm{hr}$ & $30 \mathrm{~min}$ & $2 \mathrm{hr}$ & $4 \mathrm{hr}$ \\
\hline Control & $0.612 \pm 0.014$ & $0.754 \pm 0.051$ & $0.621 \pm 0.013$ & - & - & - \\
\hline Diclofenac sodium & $0.232 \pm 0.021$ & $0.277 \pm 0.019$ & $0.277 \pm 0.027$ & $62.09 \pm 0.095$ & $63.26 \pm 0.095$ & $55.39 \pm 0.095$ \\
\hline $3 \mathbf{a}^{* *}$ & $0.403 \pm 0.024$ & $0.462 \pm 0.026$ & $0.384 \pm 0.013$ & $34.15 \pm 0.011$ & $38.72 \pm 0.010$ & $38.16 \pm 0.019$ \\
\hline $3 b^{* *}$ & $0.265 \pm 0.033$ & $0.292 \pm 0.05$ & $0.256 \pm 0.020$ & $56.69 \pm 0.033$ & $61.27 \pm 0.050$ & $58.77 \pm 0.039$ \\
\hline $3 c^{*}$ & $0.272 \pm 0.009$ & $0.341 \pm 0.041$ & $0.290 \pm 0.030$ & $55.55 \pm 0.041$ & $54.77 \pm 0.023$ & $53.30 \pm 0.013$ \\
\hline $3 d^{* *}$ & $0.366 \pm 0.043$ & $0.433 \pm 0.009$ & $0.371 \pm 0.010$ & $40.19 \pm 0.014$ & $42.57 \pm 0.063$ & $40.25 \pm 0.019$ \\
\hline $3 \mathrm{e}^{*}$ & $0.425 \pm 0.013$ & $0.526 \pm 0.019$ & $0.437 \pm 0.011$ & $30.55 \pm 0.033$ & $30.23 \pm 0.011$ & $29.62 \pm 0.024$ \\
\hline $3 f^{*}$ & $0.442 \pm 0.039$ & $0.556 \pm 0.103$ & $0.463 \pm 0.019$ & $27.77 \pm 0.134$ & $26.25 \pm 0.028$ & $25.44 \pm 0.020$ \\
\hline $3 g^{* *}$ & $0.258 \pm 0.024$ & $0.322 \pm 0.09$ & $0.271 \pm 0.17$ & $57.84 \pm 0.006$ & $57.29 \pm 0.008$ & $56.36 \pm 0.04$ \\
\hline $3 \mathbf{h}^{*}$ & $0.359 \pm 0.009$ & $0.442 \pm 0.009$ & $0.369 \pm 0.024$ & $41.33 \pm 0.039$ & $41.37 \pm 0.020$ & $40.57 \pm 0.024$ \\
\hline $3 \mathrm{i}^{* *}$ & $0.428 \pm 0.020$ & $0.524 \pm 0.024$ & $0.453 \pm 0.07$ & $30.06 \pm 0.041$ & $30.50 \pm 0.09$ & $27.05 \pm 0.009$ \\
\hline $3 \mathbf{j}^{*}$ & $0.466 \pm 0.041$ & $0.564 \pm 0.061$ & $0.471 \pm 0.011$ & $23.85 \pm 0.043$ & $25.19 \pm 0.041$ & $24.15 \pm 0.011$ \\
\hline
\end{tabular}

${ }^{*} P<0.05$ significant from control.

${ }^{* *} P<0.01$ significant from control.

TABLE 4: Result of analgesic activity.

\begin{tabular}{|c|c|c|c|c|c|c|}
\hline \multirow{2}{*}{ Compound } & \multicolumn{3}{|c|}{ Reaction time $(S)$ after drug administration (mean \pm SEM) } & \multicolumn{3}{|c|}{$\%$ Inhibition } \\
\hline & $30 \mathrm{~min}$ & $60 \mathrm{~min}$ & $90 \mathrm{~min}$ & $30 \mathrm{~min}$ & $60 \mathrm{~min}$ & $90 \mathrm{~min}$ \\
\hline Control & $2.67 \pm 0.020$ & $2.69 \pm 0.025$ & $2.70 \pm 0.03$ & - & - & - \\
\hline Diclofenac sodium & $5.31 \pm 0.03$ & $5.67 \pm 0.019$ & $5.82 \pm 0.014$ & $98.87 \pm 0.214$ & $110.78 \pm 0.217$ & $115.55 \pm 1.620$ \\
\hline $3 \mathrm{a}^{*}$ & $3.11 \pm .004$ & $3.76 \pm 0.019$ & $3.72 \pm 0.07$ & $16.47 \pm 0.019$ & $39.77 \pm 0.033$ & $37.77 \pm 0.041$ \\
\hline $3 b^{* *}$ & $5.43 \pm 0.019$ & $5.74 \pm 0.033$ & $6.11 \pm 0.010$ & $103.37 \pm 0.043$ & $113.38 \pm 0.027$ & $126.29 \pm 0.90$ \\
\hline $3 c^{*}$ & $5.34 \pm 0.041$ & $5.46 \pm 0.011$ & $5.88 \pm 0.028$ & $100 \pm 0.006$ & $102.97 \pm 0.011$ & $117.77 \pm 0.081$ \\
\hline $3 d^{* *}$ & $4.89 \pm 0.043$ & $4.66 \pm 0.010$ & $4.49 \pm 0.041$ & $83.14 \pm 0.071$ & $73.23 \pm 0.063$ & $66.29 \pm 0.038$ \\
\hline $3 \mathrm{e}^{* *}$ & $4.92 \pm 0.011$ & $5.13 \pm 0.020$ & $5.10 \pm 0.063$ & $84.26 \pm 0.023$ & $90.70 \pm 0.006$ & $88.88 \pm 0.075$ \\
\hline $3 f^{*}$ & $3.37 \pm 0.05$ & $3.22 \pm 0.008$ & $3.20 \pm 0.019$ & $26.21 \pm 0.014$ & $19.70 \pm 0.023$ & $18.51 \pm 0.010$ \\
\hline $3 \mathrm{~g}^{* *}$ & $5.35 \pm 0.023$ & $5.76 \pm 0.09$ & $5.91 \pm 0.020$ & $100.37 \pm 0.041$ & $114.12 \pm 0.020$ & $118.88 \pm 0.61$ \\
\hline $3 h^{* *}$ & $5.23 \pm 0.019$ & $5.78 \pm 0.063$ & $6.13 \pm 0.011$ & $95.88 \pm 0.05$ & $114.86 \pm 0.039$ & $127.03 \pm 0.071$ \\
\hline $3 \mathbf{i}^{* *}$ & $5.15 \pm 0.010$ & $4.88 \pm 0.014$ & $4.85 \pm 0.028$ & $92.88 \pm 0.023$ & $81.41 \pm 0.043$ & $79.62 \pm 0.91$ \\
\hline $3 j^{*}$ & $3.22 \pm 0.020$ & $3.39 \pm 0.041$ & $3.57 \pm 0.019$ & $20.59 \pm 0.008$ & $26.02 \pm 0.041$ & $32.22 \pm 0.38$ \\
\hline
\end{tabular}

${ }^{*} P<0.05$ significant from control.

${ }^{* *} P<0.01$ significant from control.

1-(4-chlorophenyl)ethanamine makes the compound potent antiproliferative agent with dual inhibition of both MCF-7 and SK-N-SH cell lines.

After carrying out the anti-inflammatory and analgesic screenings of synthesized compounds, the following conclusions are drawn. (i) Presence of $\mathrm{Br}$ and $\mathrm{NO}_{2}$ groups at 4 positions in the phenoxy nucleus contributes towards anti-inflammatory activity. (ii) Presence of tert-butyl and methoxy substituent at 4 positions does not contribute towards anti-inflammatory activity. It is also observed that compound 3c with nitro substituent in the 4 positions of phenoxy nucleus contributed effectively towards anticancer, anti-inflammatory, and analgesic activities. The analgesic activities may be due to inhibition of COX/LOX pathways which are the possible mechanisms of induction of pain.
In our future research programme, these pathways which are highly active in malignant cancerous growth would be targeted. This research would be a new breakthrough in the area of oncology where enzyme mediators and COX-2 and LOX-2 inhibitors would be utilized in suppression of tumor growth.

Scheme I. Synthesis of N-(1-(4-chlorophenyl)ethyl)-2(substituted phenoxy)acetamide derivatives (3a-e) and 2(substituted phenoxy)-N-(1-(p-tolyl)ethyl)acetamide derivatives (3f-j). Reagents and conditions: (1) ammonium carbonate and formic acid, heat very slowly till $165^{\circ} \mathrm{C} \rightarrow$ Ketone, Heat, $4-5 \mathrm{~h}, 180-185^{\circ} \mathrm{C} \rightarrow$ Reflux, concentrated hydrochloric acid, 2-3 hour, water bath $\rightarrow$ extraction, diethylether $\rightarrow$ Strongly alkaline, $30 \%$ sodium hydroxide $\rightarrow$ extraction; Diethylether (2) Chloroacetyl chloride, 10\% sodium hydroxide, 


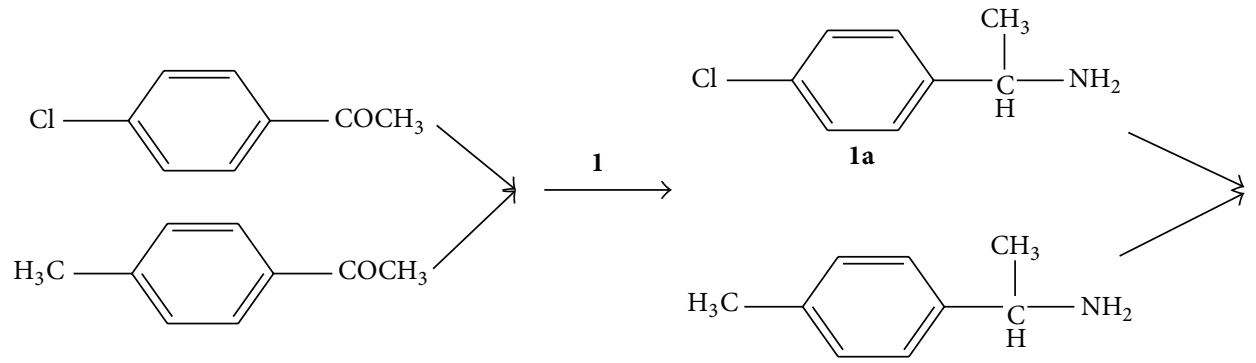

$1 b$
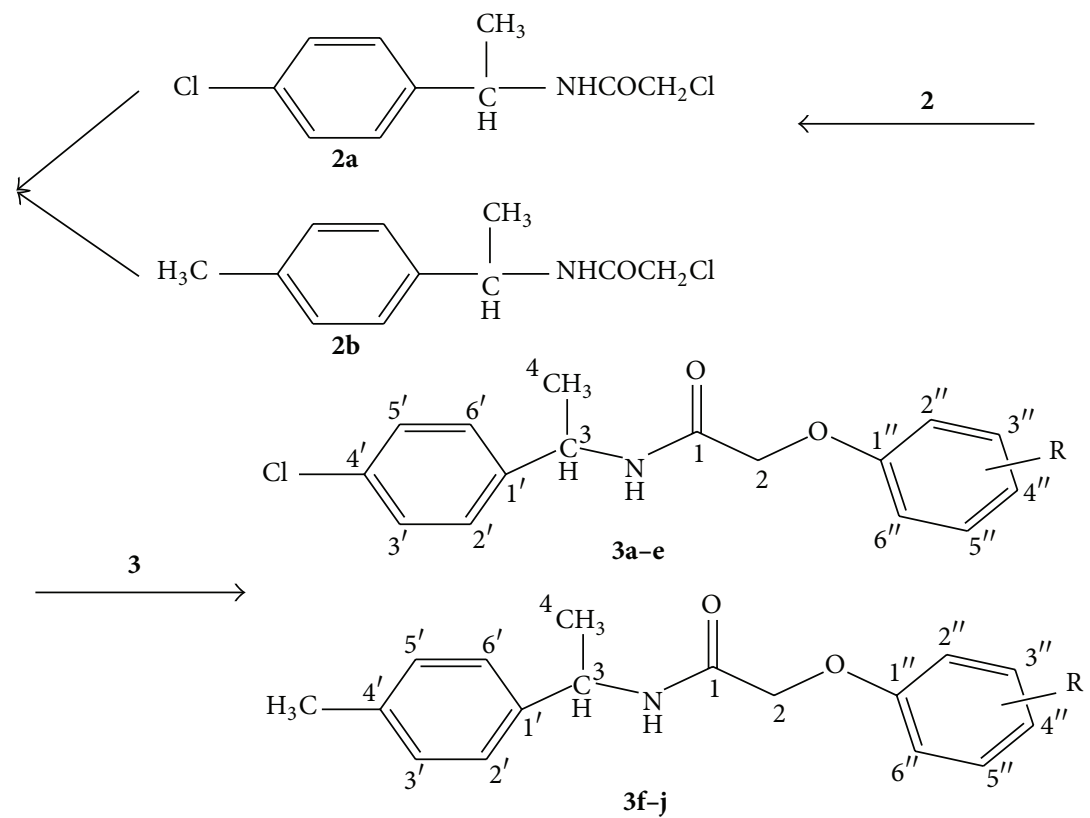

Figure 3: Synthesis of N-(1-(4-chlorophenyl)ethyl)-2-(substituted phenoxy)acetamide (3a-e) derivatives and 2-(substituted phenoxy)-N-(1(p-tolyl)ethyl)acetamide (3f-j) derivatives.

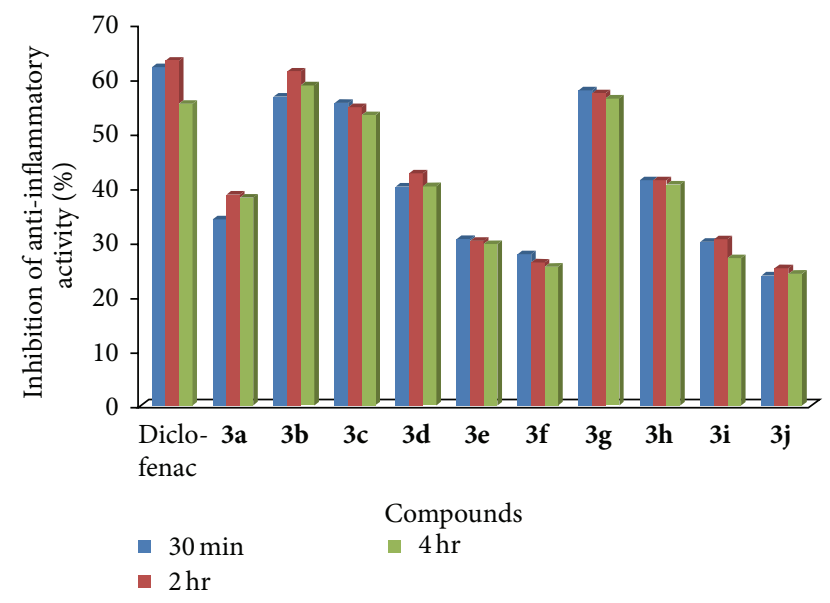

FIGURE 4: Graphical representation of (\% inhibition) of antiinflammatory activity.

ice bath, pH 9-10. (3) Substituted phenols, dry acetone, Potassium Carbonate, Potassium Iodide, Reflux.
In the series of ten compounds for activity against MCF7 (breast) cell line, compounds $\mathbf{3 b}$ and $\mathbf{3} \mathbf{c}$ showed active inhibition of the cancer cells. These two compounds were found to be highly active against the cancer cell lines. Compounds $\mathbf{3 d}, \mathbf{3 h}$, and $\mathbf{3} \mathbf{i}$ showed mild to moderate cytotoxic activities, whereas the remaining compounds were found to be completely inactive.

Out of ten compounds, $\mathbf{3} \mathbf{f}$ and $\mathbf{3 c}$ were found to be modestly active against SK-N-SH (neuroblastoma) cell line, and the remaining compounds were found to be inactive.

\section{Conflict of Interests}

The authors declare that there is no conflict of interests regarding the publication of this paper.

\section{Acknowledgment}

The authors are grateful to Professor R. M. Dubey, Vice Chancellor of IFTM University, for providing necessary facilities for this research work. 


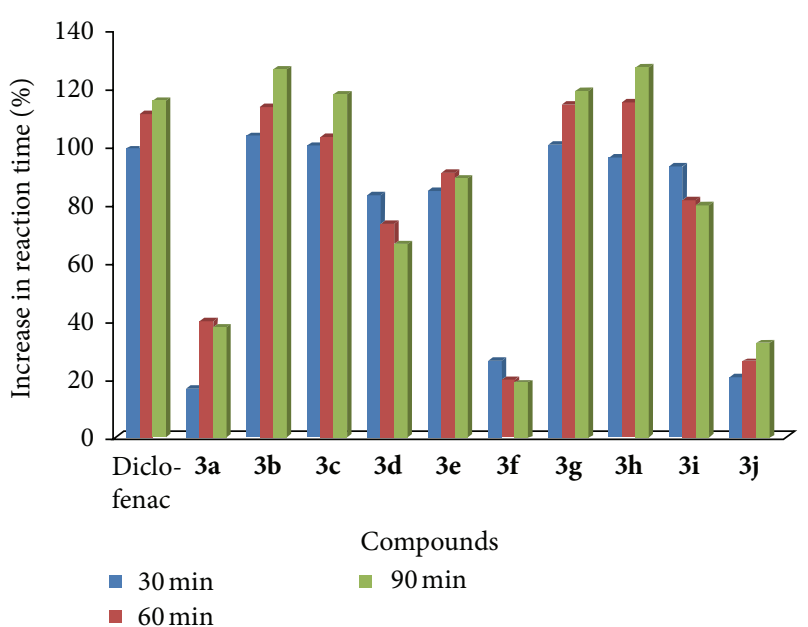

FIGURE 5: Graphical representation of \% increase in reaction time for analgesic activity.

\section{References}

[1] J. I. Johnsen, M. Lindskog, F. Ponthan et al., "Cyclooxygenase2 is expressed in neuroblastoma, and nonsteroidal antiinflammatory drugs induce apoptosis and inhibit tumor growth in vivo," Cancer Research, vol. 64, no. 20, pp. 7210-7215, 2004.

[2] M. Sonoshita, K. Takaku, M. Oshima, K. Sugihara, and M. M. Taketo, "Cyclooxygenase-2 expression in fibroblasts and endothelial cells of intestinal polyps," Cancer Research, vol. 62, no. 23, pp. 6846-6849, 2002.

[3] K. M. Sheehan, "The relationship between cyclooxygenase-2 expression and colorectal cancer," Journal of American Medical Association, vol. 282, pp. 1254-1257, 1999.

[4] M. Philip, D. A. Rowley, and H. Schreiber, "Inflammation as a tumor promoter in cancer induction," Seminars in Cancer Biology, vol. 14, no. 6, pp. 433-439, 2004.

[5] A. S. Kalgutkar and Z. Zhao, "Discovery and design of selective cyclooxygenase-2 inhibitors as non-ulcerogenic, antiinflammatory drugs with potential utility as anti-cancer agents," Current Drug Targets, vol. 2, no. 1, pp. 79-106, 2001.

[6] G. M. Pasinetti, "Cyclooxygenase and Inflammation in Alzheimers disease: experimental approaches and clinical interventions," Journal of Neuroscience Research, vol. 54, no. 1, pp. $1-6,1998$.

[7] L. M. Coussens and Z. Werb, "Inflammation and cancer," Nature, vol. 420, no. 6917, pp. 860-867, 2002.

[8] M. P. Schrey and K. V. Patel, "Prostaglandin $\mathrm{E}_{2}$ production and metabolism in human breast cancer cells and breast fibroblasts. Regulation by inflammatory mediators," British Journal of Cancer, vol. 72, no. 6, pp. 1412-1419, 1995.

[9] D. T. Hill and B. Loev, "4-(2-Thienyl)-5-methylpyrimidine. An anomalous Leuckart product," Journal of Organic Chemistry, vol. 38, no. 11, pp. 2102-2103, 1973.

[10] R. D. Bach, "Preparation of tertiary N,N-dimethylamines by the leuckart reaction," The Journal of Organic Chemistry, vol. 33, no. 4, pp. 1647-1649, 1968.

[11] L. H. Sternbach, “The benzodiazepine story," Journal of Medicinal Chemistry, vol. 22, no. 1, pp. 1-7, 1979.
[12] D. E. Thurston and D. S. Bose, "Synthesis of DNA-interactive pyrrolo[2,1-c][1,4]benzodiazepines," Chemical Reviews, vol. 94, no. 2, pp. 433-465, 1994.

[13] M. B. Gupta, R. Nath, G. P. Gupta, and K. P. Bhargava, "A study of the anti-ulcer activity of diazepam and other tranquillosedatives in albino rats," Clinical and Experimental Pharmacology and Physiology, vol. 12, no. 1, pp. 61-66, 1985.

[14] J. R. Lokensgard, C. C. Chao, G. Gekker, S. Hu, and P. K. Peterson, "Benzodiazepines, glia, and HIV-1 neuropathogenesis," Molecular Neurobiology, vol. 18, no. 1, pp. 23-33, 1998.

[15] C. Z. Ding, R. Batorsky, R. Bhide et al., "Discovery and structure-activity relationships of imidazole-containing tetrahydrobenzodiazepine inhibitors of farnesyltransferase," Journal of Medicinal Chemistry, vol. 42, no. 25, pp. 5241-5253, 1999.

[16] W. Ang, Y. Lin, T. Yang et al., "Synthesis and biological evaluation of 2-(3-Fluoro-4-nitro phenoxy)-N-phenylacetamide derivatives as novel potential affordable antitubercular agents," Molecules, vol. 17, no. 2, pp. 2248-2258, 2012.

[17] L. K. Hedstrom and B. Striepen, "IMP dehydrogenase inhibitors for treating mammalian gastrointestinal parasitic infections," WO 2007143557, 2007.

[18] A. Sun, A. Prussia, W. Q. Zhan et al., "Nonpeptide inhibitors of measles virus entry," Journal of Medicinal Chemistry, vol. 49, no. 17, pp. 5080-5092, 2006.

[19] S. K. Tipparaju, S. P. Muench, E. J. Mui et al., "Identification and development of novel inhibitors of toxoplasma gondii enoyl reductase," The Journal of Medicinal Chemistry, vol. 53, no. 17, pp. 6287-6300, 2010.

[20] K. Lee, S. H. Roh, Y. Xia, and K. W. Kang, "Synthesis and biological evaluation of phenoxy-N-phenylacetamide derivatives as novel P-glycoprotein inhibitors," Bulletin of the Korean Chemical Society, vol. 32, no. 10, pp. 3666-3674, 2011.

[21] D. Pal, S. Banerjee, and A. K. Ghosh, "Dietary-induced cancer prevention: An expanding research arena of emerging diet related to healthcare system," Journal of Advanced Pharmaceutical Technology and Research, vol. 3, no. 1, pp. 16-24, 2012.

[22] D. Pal and S. Mitra, "A preliminary study on the in vitro antioxidant activity of the stems of Opuntia vulgaris," Journal of Advanced Pharmaceutical Technology and Research, vol. 1, no. 2, pp. 268-272, 2010.

[23] A. K. Nayak, D. Pal, D. R. Pany, and B. Mohanty, "Evaluation of Spinacia oleracea L. leaves mucilage as an innovative suspending agent," Journal of Advanced Pharmaceutical Technology and Research, vol. 1, no. 3, pp. 338-341, 2010.

[24] OCED/OCDC and OECD, "Guidelines for testing of chemicals. Revised draft guidelines 423 , acute oral toxicity class method, Revised document," 2000.

[25] C. A. Winter, E. A. Risley, and G. W. Nuss, "Carrageenininduced edema in hind paw of the rat as an assay for antiiflammatory drugs," Proceedings of the Society for Experimental Biology and Medicine, vol. 3, pp. 544-547, 1962.

[26] B. B. Newbould, "Chemotherapy of arthritis induced in rats by mycobacterial adjuvant," British Journal of Pharmacology and Chemotherapy, vol. 21, pp. 127-136, 1963.

[27] N. B. Eddy and D. J. Leimbach, "Synthetic analgesics II. Dithienylbutenyl and dithienylbutylamines," Journal of Pharmacology and Experimental Therapeutics, vol. 107, no. 3, pp. 385393, 1953.

[28] V. Mathew, J. Keshavayya, V. P. Vaidya, and D. Giles, "Studies on synthesis and pharmacological activities of 3,6-disubstituted1,2,4-triazolo[3,4-b]-1,3,4-thiadiazoles and their dihydro analogues," European Journal of Medicinal Chemistry, vol. 42, no. 6, pp. 823-840, 2007. 


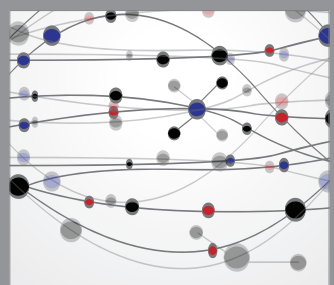

The Scientific World Journal
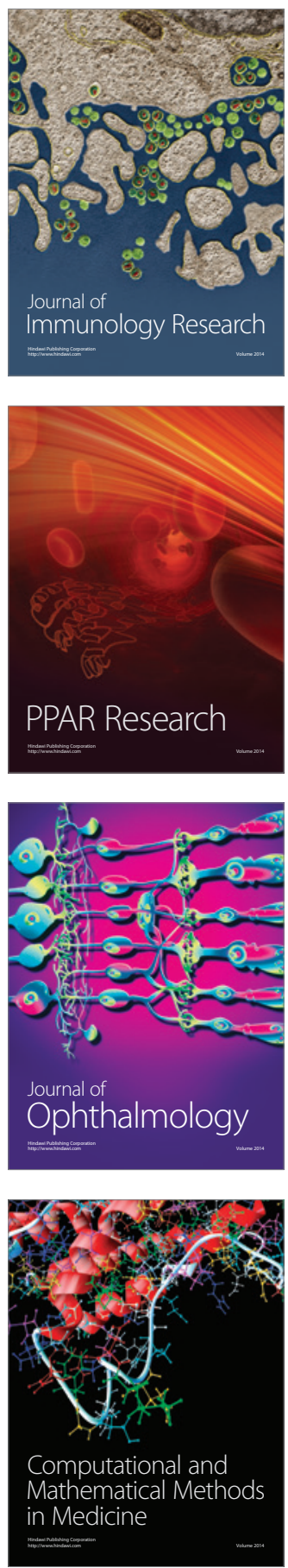

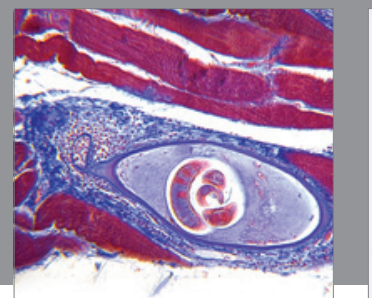

Gastroenterology

Research and Practice
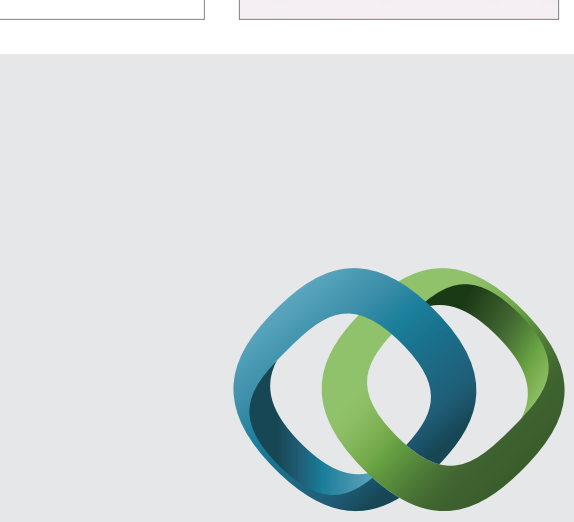

\section{Hindawi}

Submit your manuscripts at

http://www.hindawi.com
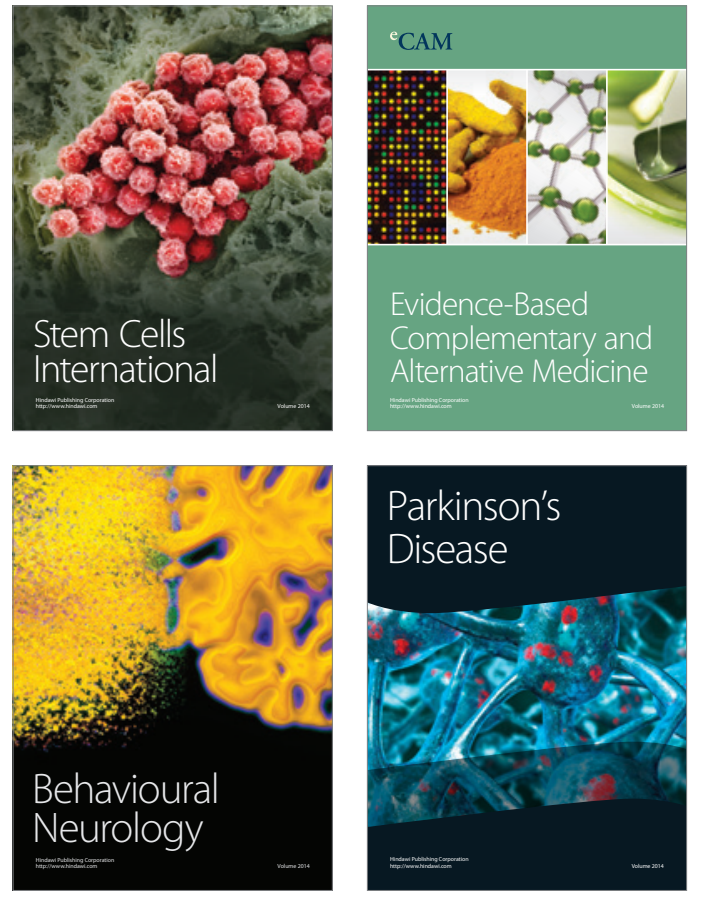
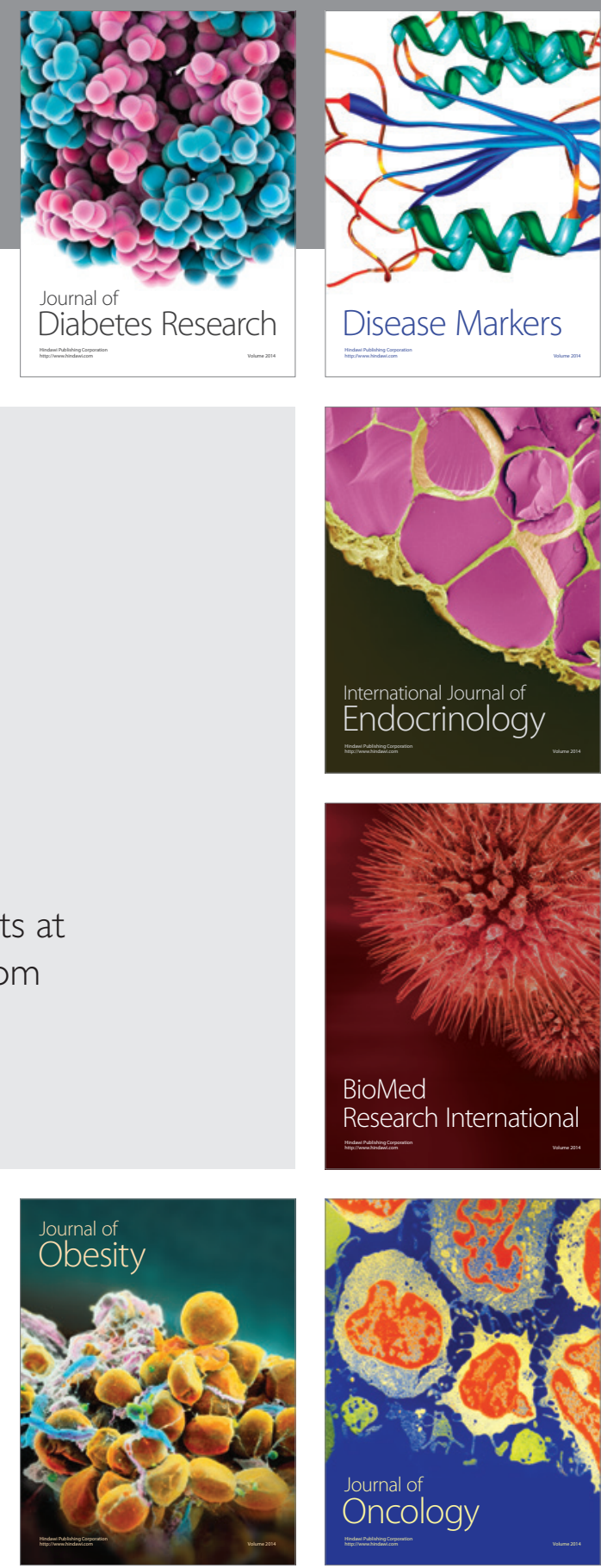

Disease Markers
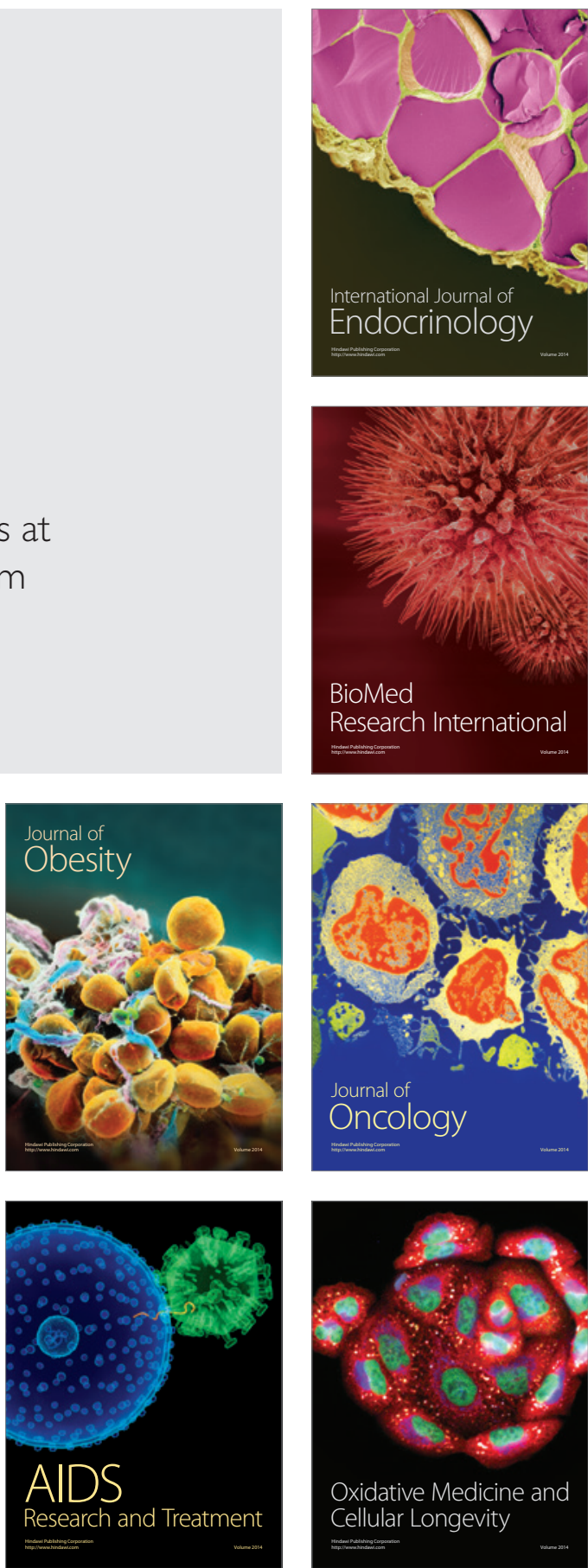